\title{
Clinical Conundrum: Dysphagia in a Patient with COVID-19 and Progressive Muscle Weakness
}

\author{
Amy Ishkanian ${ }^{1}$ (D) Alla Mehl ${ }^{1}$
}

Received: 20 August 2020 / Accepted: 22 October 2020 / Published online: 31 October 2020

(c) Springer Science+Business Media, LLC, part of Springer Nature 2020

\begin{abstract}
A 58-year-old woman with a history of diabetes presented to an acute care hospital with 6 weeks of shortness of breath, cough, and fatigue as well as a 2-week history of sudden onset of slurred speech, left upper extremity weakness, nausea/vomiting, and difficulty with ambulation. Workup was consistent with COVID-19 pneumonia and hypoxic respiratory failure. Upon admission, she required supplemental oxygen via facemask and nasal cannula, with advancement to room air. A CT of the head was unremarkable and the patient's neurological exam was largely non-focal.
\end{abstract}

On the initial Speech-Language Pathology (SLP) evaluation, she was found to have hoarse and wet vocal quality, a report of recent vomiting and overt signs of aspiration with PO (per os) trials. Nursing also reported that the patient was unable to swallow oral medication. Over the following 5 days, she developed worsening dysphagia, with poor management of oropharyngeal secretions and with reduced cough strength suspected. She required frequent self-suctioning of her oral cavity and had persistent wet, gurgly vocal quality. She was receiving nutrition via nasogastric tube. The patient was subsequently noted to develop right eye ptosis, facial weakness, and hypernasal dysarthria. Cognitive and language skills were normal.

Given worsening of the patient's symptoms, Neurology investigated various etiologies including peripheral neuromuscular weakness, myasthenia gravis, Guillain Barre Syndrome, myositis, central venous sinus thrombosis, and brainstem stroke. Initial testing, including MRI of the brain and EMG, was unrevealing. Based on repeated SLP assessments over the course of 2 weeks, the patient continued to

Amy Ishkanian

Amy.ishkanian@gmail.com

Alla Mehl

Alla.Mehl@gmail.com

1 Kings County Hospital Center, Rehabilitation Medicine C11, 451 Clarkson Avenue, Brooklyn, NY 11207, USA have severe pharyngeal dysphagia with an inability to trigger a swallow or manage her secretions.

What is causing the patient's dysphagia and what is her prognosis? How should her dysphagia be managed?

Due to a lack of improvement in swallow function at 3 weeks post-admission, a PEG was placed. Continued neuromuscular workup included total spine MRI, bilateral thigh MRI, and a thigh biopsy. Results of these tests were suggestive of myositis. In particular, the thigh biopsy result was suspicious for myositis secondary to viral infection vs idiopathic myositis with dermatomyositis. The patient was ultimately diagnosed with COVID-19-associated myositis. Four weeks after admission, the patient was transferred to Acute Rehab.

The patient continued to present with persistent pharyngeal dysphagia, however, showed some improvement in secretion management. Hyolaryngeal excursion was often absent, and the patient had persistent overt signs of aspiration with PO trials. The patient participated in dysphagia therapy including use of incentive spirometry, swallowing exercises, therapeutic PO trials, and training of oral care. Once the patient's COVID-19 test returned negative and she showed improvement in secretion management, a Modified Barium Swallow Study (MBSS) was performed 6 weeks post-admission. Results revealed severe pharyngeal dysphagia marked by diffuse pharyngeal weakness impacting hyolaryngeal excursion, UES distention, tongue base retraction, and pharyngeal stripping wave, resulting in severe pharyngeal residue and moderate-high risk of aspiration. She continued to receive feeds through the PEG. Ongoing dysphagia therapy involved respiratory muscle strength training, therapeutic PO trials, and exercises targeting hyolaryngeal excursion and pharyngeal muscle strength. A repeat MBSS was performed at 8 weeks post-admission which showed moderate pharyngeal dysphagia with reduced pharyngeal residue and more effective use of compensatory strategies, such as repeat swallows. She was started on a puree diet with thin liquids with safe swallow strategies and the PEG feeds were discontinued. The patient was discharged home with an exercise program. 


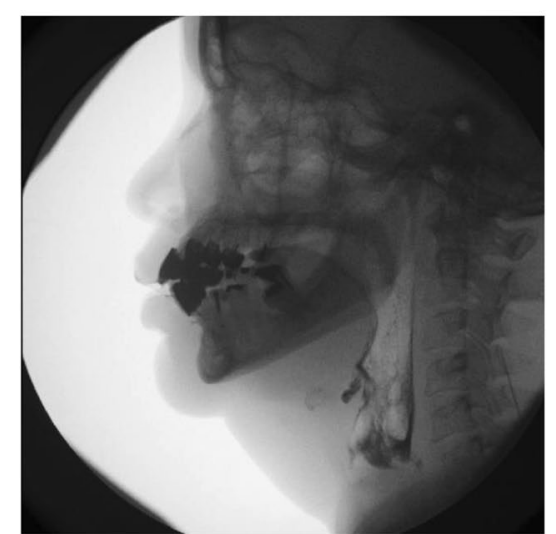

Initial MBSS showing pharyngeal residue with thin liquid after 2 repeat swallows

\section{Discussion}

Dysphagia is a common symptom of myositis, with incidence ranging between 30 and $72 \%$ of cases $[1,2]$. It is becoming apparent that myositis and other neurological manifestations can be sequelae of COVID-19 [3, 4].

Despite the patient's initial diagnosis of COVID-19, her respiratory difficulties were not severe and did not explain the extent of her dysphagia. It is necessary for SLPs to understand the typical characteristics of dysphagia caused by underlying diseases and conditions. In cases where there is an unknown etiology or the standing diagnosis does not explain the dysphagia, it is essential to manage and treat the patient's dysphagia as it presents. Furthermore, SLP assessments and input can provide crucial information that may be instrumental in leading to a diagnosis.

\section{References}

1. Claire Langdon P, Mulcahy K, Shepherd KL, Low VH, Mastaglia FL. Pharyngeal dysphagia in inflammatory muscle diseases resulting from impaired suprahyoid musculature. Dysphagia. 2012;27:408-17.

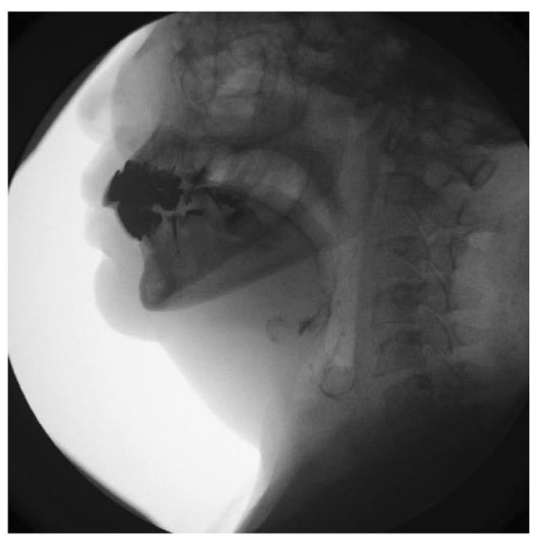

Second MBSS showing pharyngeal residue with thin liquid after 2 repeat swallows

2. Mulcahy KP, Langdon PC, Mastaglia F. Dysphagia in inflammatory myopathy: self-report, incidence, and prevalence. Dysphagia. 2012;27:64-9.

3. Benny R, Khadilkar SV. COVID 19: neuromuscular manifestations. Ann Indian Acad Neurol. 2020;23(Suppl 1):S40-2.

4. Berger JR. COVID-19 and the nervous system. J Neurovirol. 2020;26(2):143-8.

Publisher's Note Springer Nature remains neutral with regard to jurisdictional claims in published maps and institutional affiliations.

Amy Ishkanian MS CCC-SLP

Alla Mehl MS CCC-SLP, BCS-S 